\title{
Quantification of human cytoplasmic islet-cell antibodies which cross-react with mouse pancreas: a follow-up study in Type 1 (insulin-dependent) diabetic patients and in first-degree relatives
}

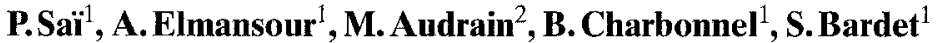 \\ ${ }^{1}$ Laboratoire d'Immuno-Endocrinologie cellulaire et moléculaire associé INRA/ENVN, Nantes, France \\ ${ }^{2}$ Laboratoire d'Immunologie biologique, Faculté de Médecine, Nantes, France
}

\begin{abstract}
Summary. We studied the heterogeneity of cytoplasmic isletcell antibodies for cross-reaction with mouse pancreas in 31 recent-onset Type 1 (insulin-dependent) diabetic patients and 31 first-degree relatives with islet-cell autoantibodies detected on human pancreas. Only six Type 1 diabetic patients displayed islet-cell antibodies binding to human pancreas but not to mouse pancreas. Among 15 first-degree relatives displaying such antibodies which did not react with mouse pancreas, including one identical twin and one subject with polyglandular autoimmunity, none developed diabetes or even lost acute insulin response to intravenous glucose after 5 years of follow-up. By contrast, 14 of $20(70 \%)$ of the Type 1 diabetic patients with islet-cell antibodies detected on human pancreas, and five first-degree relatives who progressed to a loss of acute insulin response to glucose and then to either Type 1 diabetes or glucose intolerance, also displayed antibodies reactive with mouse islets. Surprisingly, islet-cell antibodies were detectable on mouse pancreas but not on human pancreas in four Type 1 diabetic patients and in one relative who progressed to diabetes. In the five relatives who progressed to metabolic abnormalities, islet-cell antibody titres on mouse pancreas, quantified by the fluorescence intensity per islet at each serum dilution, progressively increased concomitantly with the loss of acute insulin response to glucose,
\end{abstract}

whereas islet-cell antibody titres on human pancreas remained stable. The usefulness of such quantification was also validated by the fact that antibody titres on mouse pancreas were decreased after 3 months $(p<0.01)$ in recent-onset Type 1 diabetic patients, while titres on human pancreas were not. Our results confirm that the use of mouse pancreas, combined with the conventional assay on human pancreas, reveals the heterogeneity of islet-cell autoantibodies. The presence of cross-species reactive islet-cell autoantibodies in subjects at risk may improve the predictive value, indicating relatives at lower risk whose antibodies are unable to bind to mouse islets. It could also allow the identification of subjects who progress to the disease without ever displaying islet-cell autoantibodies detectable on human pancreas. The increase of antibody titres on mouse pancreas during subject follow-up could be indicative of the worsening of the course during the prediabetic phase. Finally, islet-cell autoantibodies detected on mouse pancreas may be more transient after the onset of diabetes than is the more complex mixture of antibodies detected on human tissue.

Key words: Type 1 (insulin-dependent) diabetes mellitus, subjects at risk, cytoplasmic islet-cell autoantibodies, mouse pancreas.
Immune and metabolic abnormalities can be detected before clinical Type 1 (insulin-dependent) diabetes mellitus as markers of ongoing beta-cell destruction [1-12]. Cytoplasmic islet-cell autoantibodies (ICA) are the strongest predictive markers in subjects at risk [3, 4]. However, the predictive value of ICA is limited because many ICA positive subjects do not progress to diabetes $[11,12]$, whereas others become diabetic even though ICA have not been detected on human pancreas.

Heterogeneity of ICA has recently been established. A subset of ICA that did not cross-react with mouse pancreas may confer a low risk for progression to diabetes [13]. This subset may correspond to ICA which give a gran- ular staining restricted to human beta cells [14]. Another subset of ICA cross-reacted with mouse pancreas and may confer a higher risk [13]. It may correspond to antibodies giving a diffuse cytoplasmic staining in all human islet-cell types [14]. Heterogeneous antigenic specificities were also suggested since only some ICA positive sera from Type 1 diabetic patients were able to block binding of fluorescein-labelled immunoglobulins from patients with ICA giving a granular fluorescence pattern [15].

According to cross-reaction with mouse pancreas, we studied the cross-species heterogeneity of ICA in Type 1 diabetic patients and in first-degree relatives with regard to the predictive significance. 
End-point ICA titres often appearstable on human pancreas during the follow-up of a given subject before the clinical onset of diabetes, precluding the use of conventional ICA to indicate the possible worsening of the course. We have thus evaluated ICA titres on mouse pancreas by quantifying the mean fluorescence intensity per islet for each serum dilution of sequential samples tested with the same mouse pancreas. This method was applied to the follow-up of some first-degree relatives of Type 1 diabetic patients and validated during the follow-up of recent-onset Type 1 diabetic patients. Comparisons were made with the disappearance rate of ICA on human pancreas.

\section{Subjects and methods}

\section{Subjects}

All the subjects were included in the "Service de Diabétologie; $\mathrm{CHU}$ Nantes". Thirty-one recent-onset ( $<1$ month) Type 1 diabetic patients, in whom diagnosis of diabetes was established according to the World Health Organization criteria were studied (16 female, 15 male, mean age $28 \pm 14$ years). Thirty first-degree relatives ( $16 \mathrm{fe}-$ male, 14 male), previously classified as ICA positive on human pancreas were recruited in Nantes from our prospective evaluation of first-degree relatives of patients with Type 1 diabetes. Their mean age \pm SEM, when the first sample was drawn, was $20 \pm 4$ years. None of these relatives displayed diabetes or impaired glucose tolerance when the initial serum was drawn. Successive intravenous glucose tolerance tests (IVGTT) and oral glucose tolerance tests (OGTT) were performed as described below. Control sera for ICA determination were obtained from blood donors without personal or familial history of diabetes or of other autoimmune diseases.

\section{ICA determination on human pancreas}

ICA were determined in a blinded manner by indirect immunofluorescence on sections of a human group 0 pancreas frozen immediately after removal and kept at $-70^{\circ} \mathrm{C}$ until cryostat sections $(4-5 \mu \mathrm{m})$ were obtained. Antibody titres were determined by serial dilutions to end-point, using a fluoresceinated anti-human IgG serum (Wellcome, Dartford, Kent, UK). Results were expressed in Juvenile Diabetes Foundation (JDF) Units. However, to apply the same unit for comparison with the assay on mouse pancreas, which has not been fully standardized, results obtained on human pancreas were sometimes only expressed by end-point titres. Subjects were classified as ICA positive if they had at least two positive samples ( $\geq 5 \mathrm{JDF}$ Units). One ICA positive internal standard sample and the international reference sera accepted by the international workshops were included [16]. In the fourth international islet-cell antibody workshop, our laboratory had $100 \%$ sensitivity and $86 \%$ specificity in blinded analysis of test serum samples, with a limit of detection of $2.5 \mathrm{JDF}$ Units.

\section{ICA determination and quantification on mouse pancreas}

Mouse pancreas were obtained from 10-week-old Balb/c mice (Charles River, St Aubin les Elbeuf, France). They were snap frozen in liquid $\mathrm{N}_{2}$ and stored at $-70^{\circ} \mathrm{C}$. Serial cryostat sections $(5 \mu \mathrm{m})$ were obtained, and ICA were detected by indirect immunofluorescence. All human sera $(1 \mathrm{ml})$ were absorbed overnight at $4^{\circ} \mathrm{C}$ with $1 \mathrm{mg}$ of acetone and methanol-extracted mouse liver powder (Sigma, St Louis, Mo., USA). The sections were incubated overnight at room temperature with sera from diabetic, relative, or control subjects at serial dilutions $(1: 2$ to $1: 256)$ in $10 \mathrm{mmol} / \mathrm{l}$ phosphate buffered saline $\mathrm{pH}$ 7.2. After washing, the sections were developed with fluoresceinated rabbit $\left(\mathrm{F}(\mathrm{ab})^{\prime}\right) 2$ fragments anti-human $\mathrm{IgG}$
$(\mathrm{H}+\mathrm{L})$ (Bioatlantic, Nantes, France), for $30 \mathrm{~min}$ at room temperature. After washing, the sections were observed with a Leitz fluorescence microscope.

In order to follow-up the fluorescence obtained with sequential samples from each subject, the dilutions of each serum were tested on serial sections of the same batch of two mouse pancreases. They were then tested on sections from another batch of two pancreases on a different day. All sections were read by two independent observers who agreed to $96 \%$ without knowledge of the sample tested. Each section was scored according to three parameters: end-point titre, number of fluorescent islets and mean fluorescence intensity per islet for each serum dilution. At least 50 islets were evaluated for each dilution. The percentage of fluorescent islets was calculated for each serum dilution among the total number of islets within the two serial sections from the two batches of pancreas. The intensity of fluorescence was graded on each islet, from 0 (no staining) to 3 (maximal staining). The scores were expressed as integer values to generate the mean intensity of fluorescence \pm SEM for each serum dilution, and to allow comparison between sequential samples from a given subject. Furthermore, to take into account both variations of end-point titres and of intensities of fluorescence along the range of dilutions, an index of ICA titre was calculated as the area under the "curve of fluorescence intensity" from the 1:4 dilution to the end-point dilution. All sera were tested in at least two separate assays. In each assay, one positive intemal standard ( $80 \mathrm{JDF}$ Units on human pancreas) and one negative sample serially diluted were included as assay quality controls. The negative control was read as negative on 30 occasions. The positive control was always read as positive, with a mean end-point titre of 1/64 and an inter-assay variation of 0.5 titration steps (estimated from the SD).

\section{Metabolic study}

Acute insulin responses to i.v. glucose (AIRG) were evaluated on the morning after a 12-h overnight fast, as previously described [17]. Briefly, catheters were inserted into both antecubital veins and $0.5 \mathrm{~g} / \mathrm{kg}$ body weight of $30 \%$ glucose was injected over $2 \mathrm{~min} \pm 5 \mathrm{~s}$. Blood samples were taken 10 min prior to each bolus and at 1 and 3 min after the end of the injection. Each sample was drawn in less than $15 \mathrm{~s}$ and kept on ice until centrifugation. Plasma was then frozen at $-20^{\circ} \mathrm{C}$. Immunoreactive insulin (IRI) concentrations were measured by radioimmunoassay (SB-INSI-5; CIS International, Saclay, France) with ${ }^{125}$ I-porcine insulin, human insulin as a standard, and guinea pig antiserum to human insulin. Separation was carried out with polyethylene glycol. Undiluted plasma leading to immunoreactive insulin values above $60 \mu \mathrm{U} / \mathrm{ml}$ was further diluted until falling in the linear portion of the standard curve. Sensitivity of the assay was $2.5 \mu \mathrm{U} / \mathrm{ml}$. The intra- and inter-assay coefficients of variation were less than $10 \%$. AIRG was calculated as the sum of $1+3$ min insulin values (IRI $1+3 \mathrm{~min}$ ). Inter- and intra-individual variations of AIRG were previously reported in adult healthy control subjects [18]. A polynomial regression $\left(p<10^{-5}\right)$ was previously established between age and AIRG with a peak during puberty [19]. With this standard chart for AIRG, a "low" AIRG for each pubertal stage was defined as a value of IRI $1+3$ min below the first percentile: $29 \mu \mathrm{U} / \mathrm{ml}$ for adults and $40 \mu \mathrm{U} / \mathrm{ml}$ for Tanner's stage 4-5. OGTT ( $75 \mathrm{~g}$ glucose load for adult subjects and $1.75 \mathrm{~g} / \mathrm{kg}$ for children) were performed. Impaired glucose tolerance and diabetes mellitus were defined according to the criteria of the World Health Organization. Blood glucose was measured using a glucose oxidase method.

\section{Statistical analysis}

Statistical differences between groups were evaluated using twotailed Student's $t$-tests for paired or unpaired observations, the nonparametric Mann-Whitney tests, and the Wilcoxon's test. The chisquare test was performed to evaluate the significance of differences between percentages. Least-square linear regression was used for correlation analysis. Differences were considered significant at $p$ less than 0.05 . 


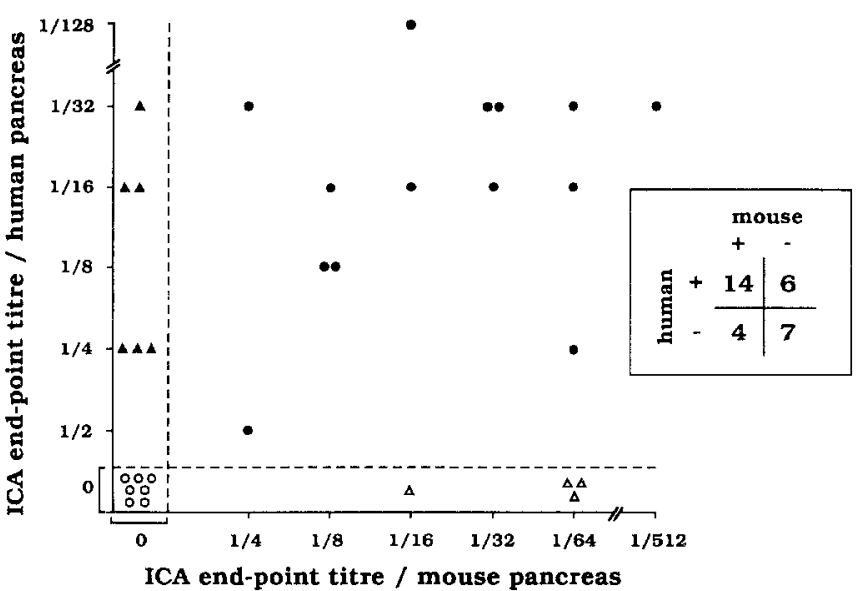

Fig. 1. Concomitant immunofluorescence detection and quantification of ICA from recent-onset Type 1 diabetic patients, using human or mouse frozen pancreas. To apply the same unit for comparison between human and mouse pancreas, results are expressed by endpoint titres. To convert results on human pancreas into JDF Units, see Subjects and methods. The number of subjects positive and negative for ICA on human or on mouse pancreas is indicated

\section{Results}

\section{ICA in Type 1 diabetic patients}

ICA were detected by indirect immunofluorescence on human pancreas in 20 of $31(65 \%)$ recent-onset Type 1 diabetic patients. Among those 20 patients, 14 (70\%) also displayed ICA cross-reactive with mouse islets, while ICA were undetectable on mouse pancreas in only six patients $(30 \%)$. Surprisingly, four out of 11 patients in whom ICA could not be detected on human pancreas, displayed ICA on mouse pancreas. Thus, the highest positivity in disease is afforded by the detection of ICA either on mouse or on human pancreas $(77 \%)$. With respect to all 31 patients, no correlation was found between ICA titres on human and on mouse pancreas (Fig.1). However, when patients who showed a total discordance between the two tests were excluded, i. e. when only those subjects were considered who displayed antibodies on human and mouse pancreases, a correlation was found between titres on both tissues $(p<0.05)$.

ICA detected on mouse pancreas quickly disappeared after the onset of diabetes. Figure 2 illustrates in two patients, that a decrease of ICA titres on mouse pancreas was generally detected by the simultaneous decrease of two parameters; end-point dilution, and mean fluorescence intensity at each serum dilution. However, in some instances, a decrease of fluorescence intensity was revealed without detectable change of end-point titre. The index of ICA titre (area under the curve of fluorescence intensity) decreased significantly for all the subjects $(p<0.01)$ as early as 3 months after the onset of the disease (Fig. 3). This contrasted with the non-significant decrease of ICA titres on human pancreas up to 1 year, as previously described [20]. This decrease was the most clear after 6 months $(p<0.001)$ when the percentage of patients without ICA on mouse pancreas was $86 \%$. A
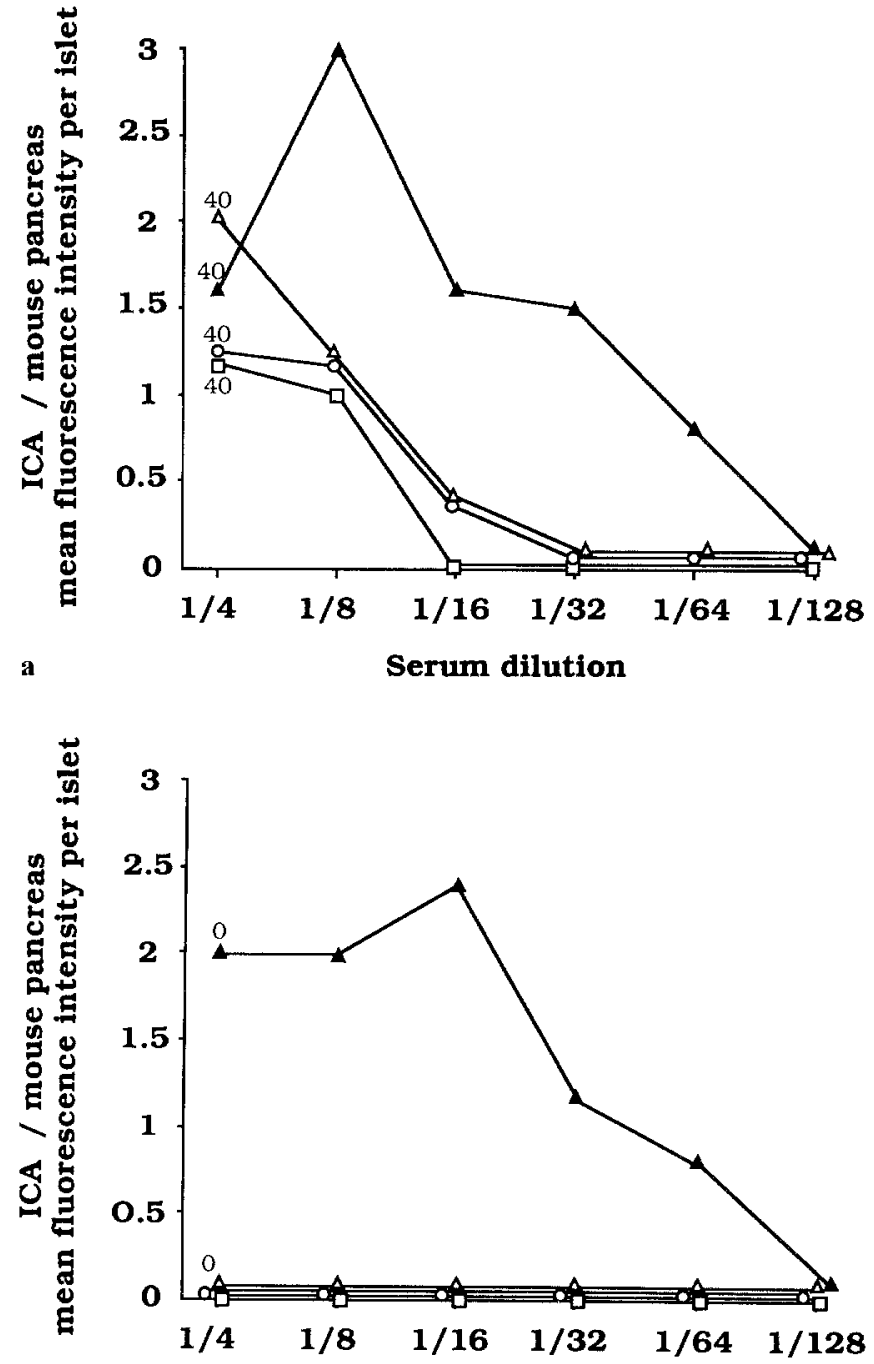

b

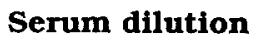

Fig.2. Typical examples of "fluorescence curves" of mouse islets as a function of serum dilutions during the follow-up of two recentonset Type 1 diabetic patients. Each panel represents one patient and reports 4 curves obtained with sera collected at entry ( $\Delta$ ), after 3 months $(\triangle), 6$ months $(O)$ and 9 months $(\square)$. Each curve is drawn by relaying points corresponding to mean readings \pm SEM of at least 50 islets (see Methods). Patient $n^{\circ} 1$ (upper panel) also displayed ICA on human pancreas, and the corresponding ICA titre on human pancreas (JDF Units) is indicated close to each curve. Patient $n^{\circ} 2$ (lower panel) had no detectable ICA on human pancreas

similar decrease $(p<0.01)$ was detected when ICA titres were expressed as the percentage of fluorescent mouse islets (data not shown).

\section{ICA in relatives of Type 1 diabetic patients}

Among 30 first-degree relatives of Type 1 diabetic patients who displayed ICA on human pancreas at entry in our prospective study, 16 did not bind at any dilution (undiluted to $1: 256$ ) to mouse islets. The remaining 14 relatives displayed ICA which cross-reacted with mouse pancreas. With respect to all 30 relatives, no correlation was found between ICA end-point titres on human and on 

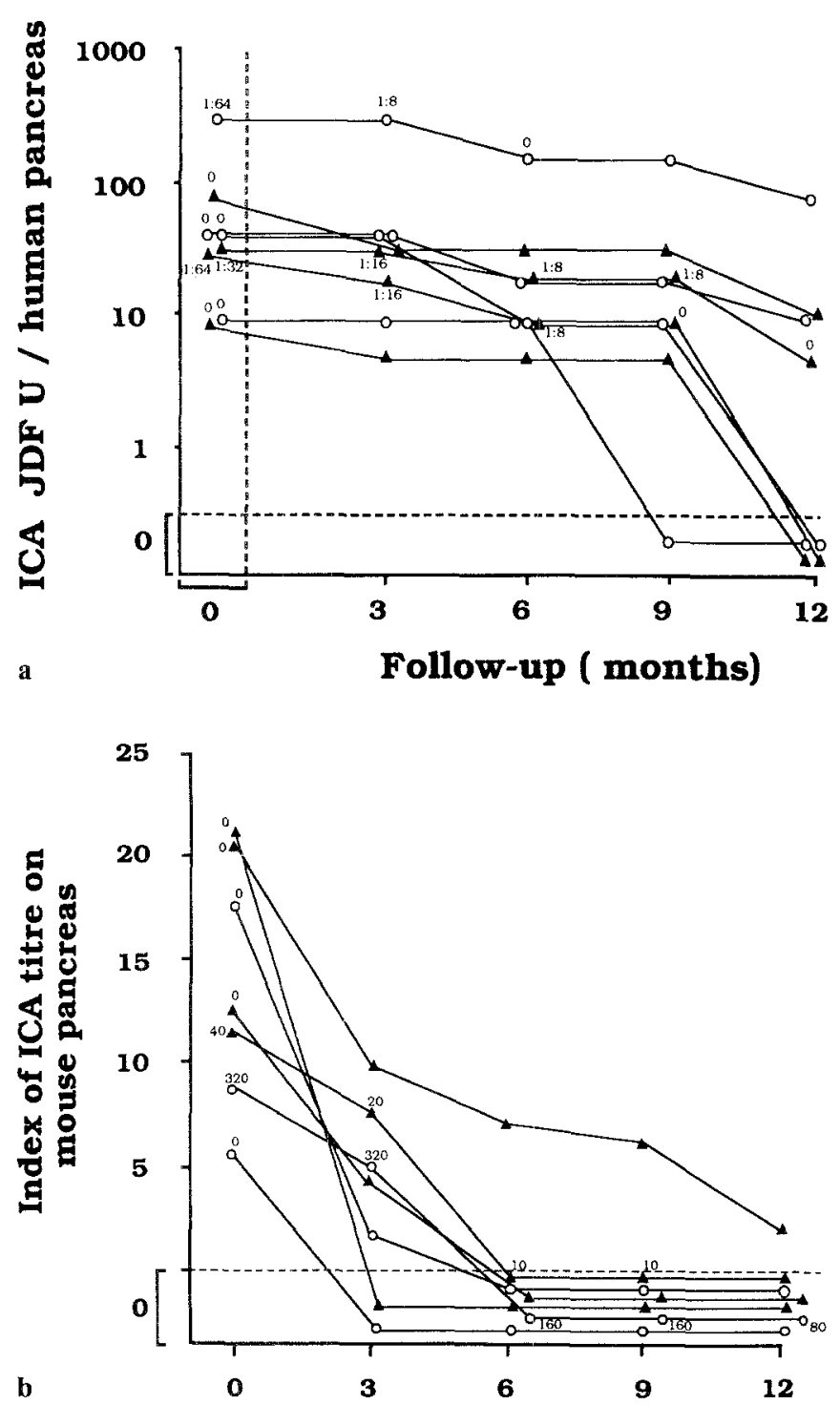

Fig. 3. Quantification of ICA on sequential serum samples from diabetic patients. ICA were quantified either into JDF Units on human pancreas (upper panel; log scale), or as the area under the "fluorescence curve" from the 1:4 dilution to the end-point dilution on mouse pancreas (lower panel). For each patient, the sequential results (at entry, and after 3,6, 9, and 12 months) are linked by a line. Close to each point, the corresponding ICA titre on the pancreas from the other species is indicated

mouse pancreas (Fig. 4). However, when only those relatives who displayed antibodies on human and mouse pancreases were considered, a correlation was found between titres on both tissues $(p<0.01)$.

The 16 relatives with ICA binding only to human pancreas at entry did not convert to cross-reactivity with mouse pancreas during testing of at least four samples corresponding to a 5-year follow-up. The AIRG of these subjects, quantified every 6 months, remained above the cutoff of the first percentile of control subjects of similar age. None of these 16 relatives progressed to diabetes. They included one non-diabetic 35-year-old identical twin (HLA DR 4/6) of a proband who had developed diabetes at the age of 15 years. This twin displayed stable ICA titres (40 JDF Units) on human pancreas over 5 years. Another

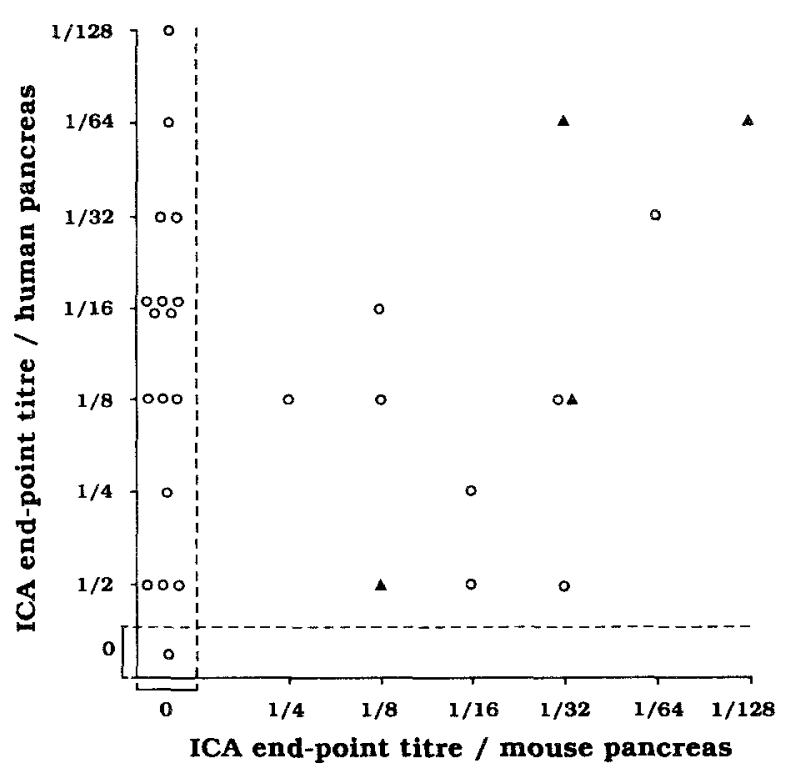

Fig.4. Concomitant detection and quantification of ICA from firstdegree relatives of Type 1 diabetic patients, using human or mouse pancreas. To apply the same measuring unit for comparison between human and mouse pancreas, results are expressed by end-point titres. To convert results on human pancreas into JDF Units, see Subjects and methods. $\boldsymbol{\Delta}$, subjects who later progressed to diabetes or to oral glucose intolerance

of these subjects was a 33-year-old women with Hashimoto's thyroiditis (DR 4/4) belonging to a family with polyglandular autoimmune diseases. She also displayed stable ICA titres on human pancreas (40 JDF Units) over 5 years.

By contrast, the AIRG of four out of the 14 relatives with ICA which cross-reacted with mouse pancreas, fell below the first percentile of control subjects (Fig.5). Two of these four subjects (one male and one female, 20 and 22 years, DR 3/4 and DR 4/4) progressed to overt diabetes after 6 and 9 months, respectively. Two other relatives (one female and one male, 20 and 18 years, DR $3 / 4$ and DR 4/4) progressed to oral glucose intolerance after 4 and 5 years, respectively.

Furthermore, clear-up variations were detectable when the sera of these four relatives were quantitated on mouse pancreas during the follow-up. Figure 6 shows typical fluorescence intensity curves as a function of dilutions from sequential serum samples in the two relatives who progressed to glucose intolerance. A progressive increase of ICA titres can be noted by the displacement of end-point titres of by the increase of fluorescence intensity. It was also reflected by an increased number of fluorescent islets (data not shown). In sequential samples during the follow-up of these relatives with ICA binding to mouse pancreas (Fig.7), a progressive increase of the index (area under the fluorescence intensity curve) contrasted with the stability of ICA titres on human pancreas. The four subjects who developed metabolic abnormalities displayed rises of 24 to $94 \%$ of this index between the initial and final samples.

Moreover, one first-degree relative (20 years, DR 3/4) of a diabetic proband, developed diabetes after 3 years of follow-up, without ever displaying detectable ICA on 


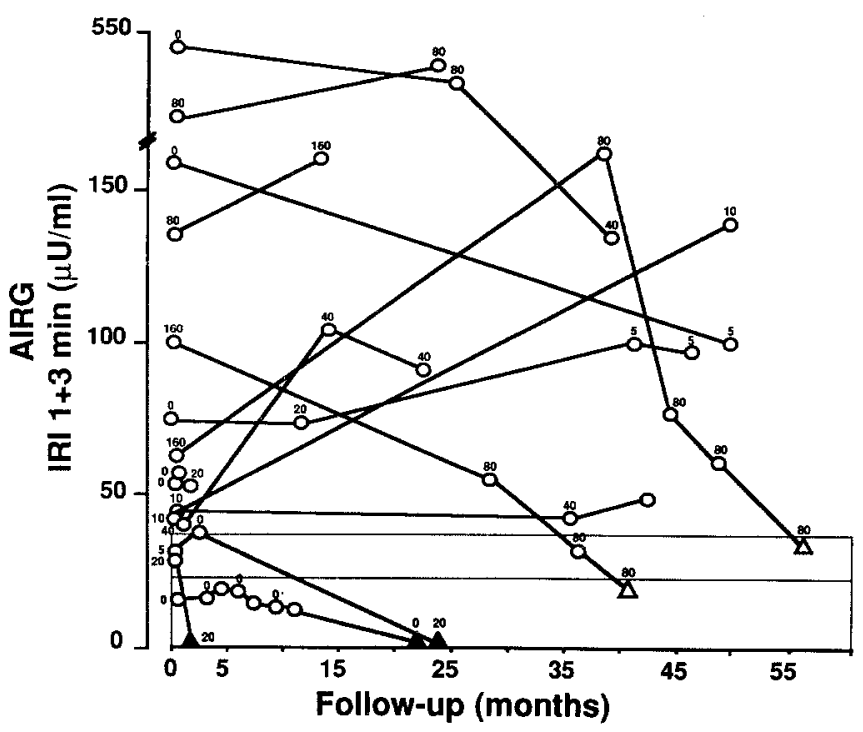

Fig.5. Sequential acute insulin responses to intravenous glucose (AIRG) in relatives tested for ICA on mouse pancreas. Sequential AIRG (IRI 1 + 3 min) from each subject are linked by lines. Subjects who did not develop diabetes $(O)$ and those who progressed to diabetes $(\boldsymbol{\Delta})$ or to glucose intolerance $(\Delta)$ are indicated. The two horizontal lines indicate the first percentile of control AIRG for pubertal Tanner's stages 4 and $5(40 \mu \mathrm{U} / \mathrm{ml})$ and for young adults $(25 \mu \mathrm{U} / \mathrm{ml})$. For each AIRG value, the corresponding ICA titre on human pancreas (JDF Units) is indicated
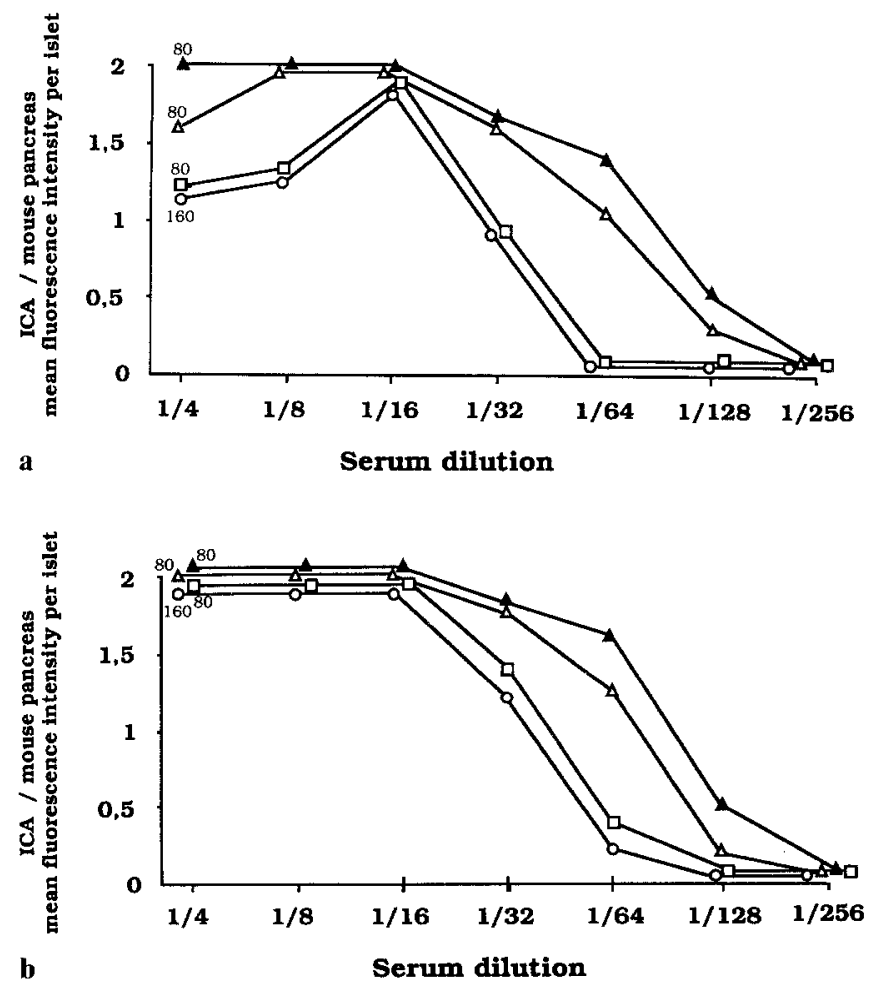

Fig.6. Examples of "fluorescence curves" of mouse islets as a function of serum dilutions from two relatives who progressed to glucose intolerance. Each panel gives the curves obtained with sequential sera collected during the follow-up. Upper panel: subject $n^{\circ} 1$ at entry $(O)$ and after 37 months $(\square), 45$ months $(\triangle)$, and 56 months $(\Delta)$. Lower panel: subject $\mathrm{n}^{\circ} 1$ at entry $(O)$ and after 27 months ( $\square$ ), 35 months $(\Delta)$, and 40 months $(\Delta)$. Each curve is drawn by relaying points corresponding to mean readings \pm SEM of at least 50 islets. For each of these sequential samples, the titre on human pancreas (JDF Units) is given on the left of the curve

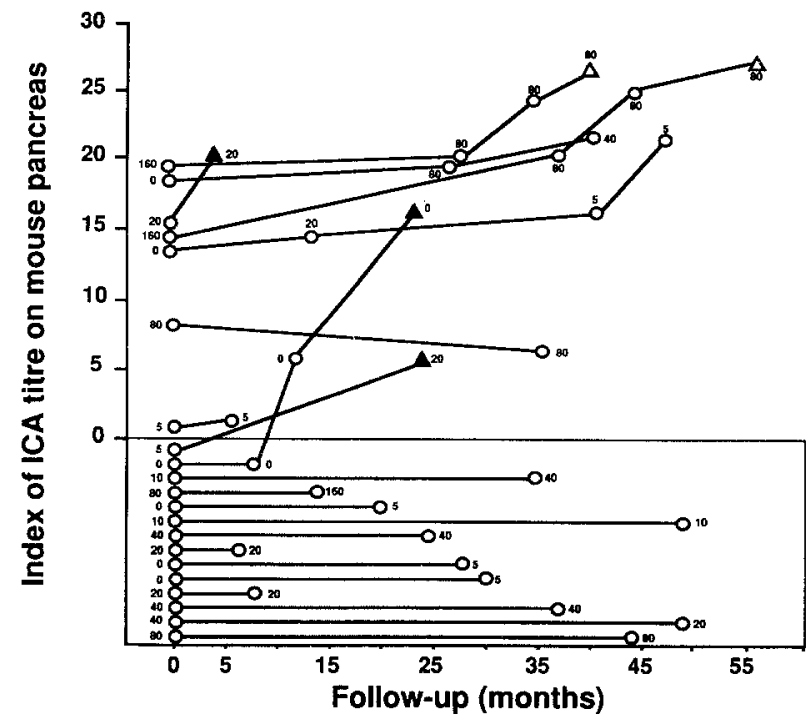

Fig. 7. Index of ICA titre on mouse pancreas during the follow-up of some relatives. The index of ICA titre is calculated as the area under the "fluorescence curve" of mouse islets (see Subjects and methods). Sequential results for each subject are linked by lines. Subjects who did not develop diabetes $(O)$ and those who progressed to diabetes $(\Delta)$ or to glucose intolerance $(\triangle)$ are indicated. Close to each point, the corresponding ICA titre on human pancreas (JDF Units) is indicated

human pancreas (seven sequential samples). His AIRG remained blunted during seven IVGTT until overt diabetes (Fig. 5). When tested on mouse pancreas, no ICA were detected over 2 years (three sequential samples), but they appeared 1 year before the onset of diabetes and increased in the following sample ( 6 months before the onset of the disease) (Fig.7).

\section{Discussion}

According to cross-reaction with mouse pancreas, our study reinforces previous findings concerning ICA heterogeneity [13]. At least two subsets of ICA were found in Type 1 diabetic patients and in first-degree relatives: ICA which bound to human pancreas but not to mouse pancreas, and ICA which cross-reacted with mouse pancreas. In addition, ICA were detected on mouse pancreas in some Type 1 diabetic patients without ICA detectable on human pancreas, and in one first-degree relative who progressed to diabetes without ever displaying ICA on human pancreas.

ICA which do not cross-react with mouse islets may be indicative of a low risk of progression to diabetes [13]. They may correspond to ICA giving a granular staining mainly restricted to beta cells and most likely directed against glutamate decarboxylase $[14,21]$ identified as the $64 \mathrm{kDa}$ antigen [22]. ICA which cross-react with mouse pancreas could confer a higher risk [13]. They may correspond to antibodies which give diffuse cytoplasmic staining in all islet-cell types [1], and their targets could be glycolipids [23]. In our study, all relatives who progressed to metabolic abnormalities, as well as most Type 1 diabetic patients, displayed ICA which reacted with 
mouse pancreas. Those ICA may result from a break of immune self-tolerance to several non-species-specific islet molecules. Conversely, fewer diabetic patients displayed ICA which reacted only with human pancreas, and none of the ICA positive relatives with this pattern, including an identical twin [24] and a subject with polyglandular autoimmunity [25], developed diabetes. Those ICA only detectable on human pancreas may result from a break of self-tolerance only to beta-cell molecules which rarely leads to the disease. The presence of such ICA that do not cross-react with mouse pancreas may even reflect a protective immune response characterized by an increased frequency of protective HLA haplotypes $[13,26]$.

The predictive value of ICA detected on human pancreas becomes greater as their titres increase $[3,4]$. However, a high positivity threshold only identifies some of the subjects who progress to the disease. A lower threshold is desirable to detect more patients, but this would require combining the ICA assay on human pancreas with other tests in order to enhance predictive value. Determination of ICA binding to mouse pancreas may improve this predictive value, lowering the "practical cut-off" for ICA positivity on human pancreas, and indicating relatives without mouse cross-reactive ICA. It may also indicate some subjects without ICA on human pancreas who nevertheless progress to diabetes. Our data suggest that some individuals who develop diabetes without displaying ICA on human pancreas may be incorrectly classified as ICA negative when only human tissue is used. ICA which only react with mouse pancreas may, in normal human islets, correspond to epitopes either hidden or expressed below the detection limit when titres are very low. Although human sera in our study were absorbed by mouse liver powder, we cannot exclude that this reaction with mouse but not with human pancreas may have been due to the presence of heterophilic antibodies.

Quantification of ICA on mouse pancreas indicated that ICA titres increased progressively, in conjunction with a loss of acute insulin response to glucose, in all firstdegree relatives who developed metabolic abnormalities. This increase contrasted with the stability of ICA titres on human pancreas, and even occurred in a relative who developed diabetes without ever displaying ICA on human pancreas. Sequential quantification of ICA on mouse pancreas may thus be indicative of a worsening of the course during the prodromal phase. The value of quantifying ICA on mouse pancreas was also validated by the follow-up of recent-onset diabetic patients. In these subjects, ICA titres after 3 months were already decreased on mouse pancreas but not on human pancreas. Although earlier detection of decreased ICA titres on mouse pancreas may have been due to more accurate quantification, it is possible that ICA which cross-react with mouse pancreas are more transient and more closely related to the onset of the disease than is the more complex mixture of ICA detected on human pancreas.

Acknowledgements. We are deeply indebted to Professor C. Boitard for very helpful discussions, and for the confirmation of ICA titres in diabetic patients. We are also grateful to all members of the GOFE-
DI (Groupe Ouest-France pour l'Etude du Diabète Insulino-dépendant). We thank E. Gouin for producing the figures. This work was supported by grants from INSERM (Réseau de recherche clinique), Caisse Régionale d'Assurance Maladie de Loire-Atlantique, INRA, and Ministère de l'Agriculture et de la forêt.

\section{References}

1. Castano L, Eisenbarth GS (1990) Type 1 diabetes: a chronic autoimmune disease of man, mouse and rat. Ann Rev Immunol 8: 647-679

2. Maclaren NK (1988) How, when and why to predict IDDM. Diabetes 37: 591-594

3. Bonifacio E, Bingley PJ, Shattock M et al. (1990) Quantification of islet-cell antibodies and prediction of insulin-dependent diabetes. Lancet 335: 147-149

4. Tarn AC, Thomas JM, Dean BM et al. (1988) Predicting insulindependent diabetes. Lancet I: 845-850

5. Baekkeskov S, Landan M, Kristensen JK et al. (1990) Antibodies to a $64,000 \mathrm{M}_{\mathrm{s}}$ human islet-cell antigen precede the clinical onset of insulin-dependent diabetes. J Clin Invest 79: 93-97

6. Vardi P, Crisa L, Jackson RA et al. (1991) Predictive value of intravenous glucose tolerance test insulin secretion less than or greater than the first percentile in islet-cell antibody positive relatives of type 1 (insulin-dependent) diabetic patients. Diabetologia 34: 93-102

7. Bleich D, Jackson RA, Soeldner JS, Eisenbarth GS (1990) Analysis of metabolic progression to type 1 diabetes in islet-cell antibody positive relatives of patients with type 1 diabetes. Diabetes Care 13: 111-118

8. Srikanta S, Ganda OP, Rabizadeh A, Soeldner JS, Eisenbarth GS (1985) First-degree relatives of patients with type 1 diabetes mellitus: islet-cell antibodies and abnormal insulin secretion. $\mathrm{N}$ Engl J Med 313: 461-464

9. Riley WJ, Maclaren NK, Krischer J et al. (1990) A prospective study of the development of diabetes in relatives of patients with insulin-dependent diabetes. N Engl J Med 323: 1167-1172

10. Bruining GJ, Molenaar JL, Grobbe DE et al. (1989) Ten-year follow-up study of islet-cell antibodies and childhood diabetes mellitus. Lancet I: 1100-1103

11. Bosi E, Becker F, Bonifacio E et al. (1991) Progression to type 1 (insulin-dependent) diabetes in autoimmune endocrine patients with islet-cell antibodies. Diabetes 40:977-984

12. Landin-Olsson M, Karlsson A, Dahlquist G, Blom L, Lernmark A, Sundkvist G (1989) Islet-cell and other organ specific autoantibodies in all children developing type 1 (insulin-dependent) diabetes mellitus in Sweden during one-year and in matched control children. Diabetologia 32: 387-395

13. Gianani R, Pugliese A, Bonner-Weir S et al. (1992) Prognostically significant heterogeneity of cytoplasmic islet-cell antibodies in relatives of patients with type 1 diabetes. Diabetes 41: $347-353$

14. Genovese S, Bonifacio E, Dean BM, McNally J, Bosi E, Bottazzo GF (1992) Distinct cytoplasmic islet-cell antibodies with different risk for type 1 (insulin-dependent) diabetes mellitus. Diabetologia 35: $385-388$

15. Timsit J, Caillat-Zucman S, Blondel H, Bach JF, Boitard C (1992) Islet-cell antibody heterogeneity among type 1 (insulin-dependent) diabetic patients. Diabetologia 35:792-795

16. Bonifacio E, Lernmark A, Dawkins RL et al. (1988) Serum exchange and the use of dilutions have improved precision of measurement of islet-cell antibodies. J Immunol Methods 106: 83-88

17. Bardet S, Rohmer V, Maugendre D et al. (1991) Acute insulin response to intravenous glucose, glucagon and arginine in some subjects at risk for type 1 (insulin-dependent) diabetes mellitus. Diabetologia 24: 648-654

18. Bardet S, Pasqual C, Maugendre D, Remy JP, Charbonnel B, Saï P (1989) Inter and intra individual variability of acute insulin response during intravenous glucose tolerance tests. Diabete Metab 15:224-232 
19. Joseph MG, Bardet S, Stetieh H et al. (1990) Age-related changes of acute insulin response to IV glucose (AIRG) in subjects at risk for type 1 diabetes and progression to diabetes. Diabetologia 33 [Suppl]: A183 (Abstract)

20. Boitard C, Feutren G, Castano L et al. (1987) The effect of cyclosporin A treatment on the production of antibody in insulindependent (type 1) diabetic patients. J Clin Invest 80: 1607-1612

21. Maclaren N, Kaufman D, Atkinson MA et al. (1992) A subset of islet-cell cytoplasmic autoantibodies in insulin dependent diabetes are reactive to glutamate decarboxylase (GAD). Diabetes 14 [Suppl]: A150 (Abstract)

22. Baekkeskov S, Aanstoot HK, Christgau S et al. (1990) Identification of the $64 \mathrm{~K}$ autoantigen in insulin-dependent diabetes as the GABA-synthesizing enzyme glutamic acid decarboxylase. Nature 347: 151-156

23. Colman PG, Nayak RC, Campbell IL, Eisenbarth GS (1988) Binding of cytoplasmic islet-cell antibodies is blocked by human pancreatic glycolipid extracts. Diabetes 37: 645-652

24. Pyke DA (1988) Development of diabetes in identical twins. Adv Exp Med Biol 246: 255-258
25. Bottazzo GF, Florin-Christensen A, Doniach D (1974) Islet-cell antibodies in diabetes mellitus with autoimmune polyendocrine deficiencies. Lancet 2: 1279-1283

26. Erlich HA, Griffith RL, Bugawan TL, Ziegler R, Alper C, Eisenbarth GS (1991) Implication of specific DQB1 alleles in genetic susceptibility and resistance by identification of IDDM siblings with novel HLA-DQB1 allele and unusual DR2 and DR1 haplotypes. Diabetes 40:478-481

Received: 4 January 1993

and in revised form: 30 March 1993

Dr. P.Saï

Laboratoire d'Immuno-Endocrinologie cellulaire et moléculaire Faculté de Médecine (3è étage)

1 rue Gaston Veil

F-44035 Nantes

France 